\title{
Distributed and Dynamic Map-less Self-reconfiguration for Microrobot Networks
}

\author{
Hicham Lakhlef, Hakim Mabed, and Julien Bourgeois \\ UFC/FEMTO-ST, UMR CNRS 6174, 1 cours Leprince-Ringuet, Montbeliard, France \\ \{hlakhlef,hmabed,julien.bourgeois\}@femto-st.fr
}

\begin{abstract}
MEMS microrobots are low-power and lowmemory capacity devices that can sense and act. One of the most challenges in MEMS microrobot applications is the self-reconfiguration, especially when the efficiency and the scalability of the algorithm are required. In the literature, if we want a self-reconfiguration of microrobots to a target shape consisting of $P$ positions, each microrobot should have a memory capacity of $P$ positions. Therefore, if $P$ equals to millions, each node should have a memory capacity of millions of positions. Therefore, this is not scalable. In this paper, nodes do not record any position, we present a self-reconfiguration method where a set of microrobots are unaware of their current position and do not have the map of the target shape. In other words, nodes do not store the positions that build the target shape. Consequently, memory usage for each node is reduced to $O(1)$. An algorithm of self-reconfiguration to optimize the communication is deeply studied showing how to manage the dynamicity (wake up and sleep of microrobots) of the network to save energy. Our algorithm is implemented in Meld, a declarative language, and executed in a real environment simulator called DPRSim.
\end{abstract}

Keywords-distributed algorithms; DiMEMS; MEMS microrobot; self-reconfiguration; physical topology; optimization; mobility; dynamicity; energy

\section{INTRODUCTION}

Micro-electro-mechanical systems (MEMS) is a technology that enables the batch fabrication of miniature mechanical structures, devices and systems that can sense and act. Their applications require a massive deployment of nodes, thousands or even millions [16] which will give birth to the Distributed Intelligent MEMS (DiMEMS) [3].

Microrobots systems have a wide range of applications such as odor localization, firefighting, medical service, surveillance, search, rescue, and security. To do these tasks the nodes have to perform the self-reconfiguration. Selfreconfiguration can be seen from two different points of view. First, it can be defined as a protocol, centralized or distributed, which transforms a set of nodes to reach the optimal logical topology from a physical topology [7], e.g, the chain represents the worst complexity case with $O(n)$, the square represents the best one with $O(\sqrt{n})$. On the other hand, the self-reconfiguration is built from modules which are autonomously able to change the way they are connected, thus changing the overall shape of the network. This process is difficult to control, because it involves the distributed coordination of a large numbers of identical modules connected in time-varying ways. Optimizing the energy cost of self-reconfiguration algorithms has a direct impact on the energy efficiency of any swarm.

Mobility and dynamicity of the system are making the problem even harder to handle as the logical topology of the system has to be stored in a distributed structure, as the spanning tree. The range of exchanged information and the number of movements determine the communication and the energy complexity of the distributed algorithm. When the information exchange involves close neighbors, the complexity is moderate and the resulting distributed selfreconfiguration scales gracefully if the algorithm does not need the predefined positions of the target shape.

This work takes place within the Claytronics project and aims at optimizing the logical topology of the network.

\section{RELATED WORKS}

Many terms refer to the concept of self-reconfiguration. The self-organization term can be found in protocols for sensors networks to form a sphere or a polygon from a center node [12], [13]. The term redeployment is also a new term to address self-reconfiguration for sensor networks [8], [5]. For self-reconfiguration with robots or microrobots, there is the protocol in [15] where the desired configuration is grown from an initial seed module. A generator uses a 3D CAD model of the target configuration and outputs a set of overlapping blocks which represent this configuration. In the second step, this representation is combined with a control algorithm to produce the final self-reconfiguration algorithm. Among the centralized algorithms we find centralized selfassembly and/or reconfiguration algorithms [14]. Other approaches give each node a unique ID and a predefined position in the final structure; see for instance [17]. The drawback of these methods is the centralized paradigm and the need for nodes identification. More distributed approaches in [6], [9], [10], [11]. Claytronics, is a project led by Carnegie Mellon University and Intel corporation. In Claytronics, microrobots called catoms (Claytronics atoms. The idea is to have hundreds of thousands of microrobots forming by self-reconfiguration together objects of any shape or size. Many works have already been done within the Claytronics project. In [4], the authors propose a metamodel for the reconfiguration of catoms starting from an initial configuration to achieve a desired configuration using creation and destruction primitives. The authors use these two functions to simplify the movement of each catom. In 
[2], a scalable protocol for Catoms self-reconfiguration is proposed, written with the MELD language [1] and using the creation and destruction primitives. In all these works, the authors assume that all Catoms know the correct positions composing the target shape at the beginning of the algorithm.

\section{Contributions}

In this paper, we propose a new distributed approach for self-reconfiguration of MEMS microrobots, where the target form is built incrementally, and each node in the current increment acts as a landmark for other nodes to form the next increment, which will belong to the form. We introduce a state model where each node can see the state of its physical neighbors to achieve the self-reconfiguration, using the states the nodes collaborate and help each other. In this paper each node predicts its future actions (movements), so it can compute the energy amount that will spend before the beginning of the algorithm. The prediction property makes the algorithm robust and energy-aware, because the node can make sure that it has correctly followed the algorithm and it is aware of the amount of energy that it will use. Also, to keep the energy and to augment the probability that the node will finish its task, each node is aware of the time slots where it can sleep to save energy.

In the proposed algorithm, the exchange of messages is limited to the construction of the spanning tree. The spanning tree is used to ensure the connectivity of the network and dynamically manage the nodes that can move. Contrary to existing works, in our algorithm each node has no information on the correct positions (predefined positions) of the target shape, the algorithm does not need to know the network size (nodes number) and movement of microrobots is fully implemented.

We propose an efficient distributed algorithm for nodes self-reconfiguration where each node moves by rotation around their physical neighbors. We study the case of a selfreconfiguration from a chain of microrobots to a square. The performance of the self-organization algorithm is evaluated according to the number of rotations and the time taken. In this paper the MEMS network is organized initially as a chain. By choosing a straight chain as the initial shape, we aim to study the performance of our approach in extreme case. Indeed, the chain form represents the worst physical topology for many distributed algorithms in terms of fault tolerance, propagation procedures and convergence. Indeed, the number of direct contacts between macro-robots is minimal and secondly the average distance between two robots (in terms of number of hops) is of $(n+1) / 3$ where $n$ is the number of robots.

We present the results of the simulations made with the declarative language Meld [1] and the open-source simulator DPRSim [18].

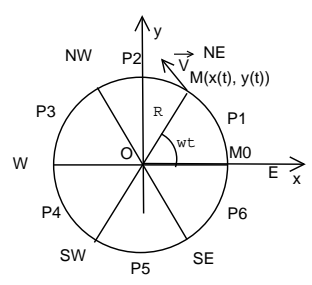

Figure 1. Node modeling, in each movement the node travels the same distance.

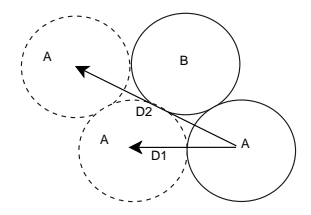

Figure 3. Traveled distance in one movement $=2 \mathrm{R}$, the node $\mathrm{A}$ travels $2 \mathrm{R}$ in one movement

\section{Model And Definitions}

Within Claytronics, a catom (figure 2) called in this paper a node is modeled as a sphere which can have at most six neighbors. Each node is able to sense the direction of its physical neighbors (east (E), west (W), north-east (NE), south-east (SE), south-west (SW) and north-west (NW)). In this work, the starting physical topology is a chain of $n$ nodes linked together. A chain is a connected set of nodes where each node has two neighbors excepting the two extremities having one neighbor. We will take the example of nodes that have neighbors in NW and SE directions and we will show after how to generalize. A node $A$ is in neighbor's list of node $B$ if $A$ is physically linked to $B$. In Claytronics, communications are only possible through contact (i.e. only neighbors can have a direct communication).

Snap-Connectivity: A dynamic network ensures a SnapConnectivity, if in all rounds of the algorithm the network is connected (there is always a path between two nodes).

Spanning tree: Is a graph composed of all nodes without any cycle. In the spanning tree, a node is either a child or a parent, a leaf is node without children.

We call the own movements of a given node the number of movements performed by. Consider the figure 1 which represents a microrobot. We say that a microrobot has done a single movement if the distance between its former position and its new position is exactly twice the radius $D 1=2 R$. For example, if the node is in a position at a distance $D 2$ (see the figure 3 ) from the former position it has done two movements. We assume (and according to the simulation tools) that the change of message (consultation) between two physical neighbors is carried without complexity $(0$ message), while the distance between them is zero. If a node to decide needs to know the state of a non-physical neighbor message exchange is required. 


\section{PROposed PROTOCOL}

\section{A. Dynamic Algorithm with Safe Connectivity (DASC)}

In DASC, each node can only move around its physical neighbor. To ensure snap-connectivity only nodes that do not cause network non-connectivity can move around neighbors. For this purpose we introduce the use of the tree to dynamically manage the leaf nodes authorized to move.

Description of the algorithm

The algorithm runs in rounds. In each round, satisfied predicates are executed. In a current round predicates with best priority are executed while others with lowest priority are ignored. We notice that in DASC, the state change actions, represented by predicates labeled $\mathrm{P} 1$, are considered more prior than a movement actions represented by P2. The distributed algorithm seeks the desired form by an incrementally process. In a completed increment, the nodes that build it belong already to the form. The initiator which is the root initializes the tree and becomes a parent of itself (5). A node if it does not have a parent becomes a child of one of the neighbor parents (6), a node is a leaf if all its neighbors are parents (7). At the beginning all nodes are initialized with the bad state with predicate (2). The initiator belongs to the target shape, so it changes its state to $\operatorname{good}(3)$, it will help its neighbors or future neighbors to take correct positions. The nodes already in the target shape act as a benchmark to neighbor or future neighbor nodes to complete a new layer. The nodes already in the form change their states with the predicate (3) and (8) and they become constant, the node can check if its neighbor have the good state with the predicate (3) and (8). The node that starts the move is the lowest node in the chain that is the first leaf of the first tree built, it rises until the root using motion around other nodes with predicates (11) and (12). The nodes of the current layer (layer being built) may make motion either at left directly or NW directly with the last three predicates. The node can change its state to good with predicate (3) if it cannot move to left or in NW. With the predicate (13) the node moves at left, it will have the neighbor that used it to move at NE direction, it repeats the same motion until it arrives to the diagonal node that have the state spe, it cannot move around this last only if the diagonal node has not a neighbor node in the $\mathrm{E}$ direction. Diagonal nodes take the state spe with the predicate (4) and (10), and with (14) the node moves until it takes a correct position. The state change has a priority as the moving actions to avoid bad motion, because of this we introduce the priority in our algorithm. To avoid message exchange the node can change its state to good if it has 3 neighbors having the state good (9) or one neighbor has spe state and has neighbors in the both $\mathrm{NE}$ and NW directions with predicate (10).

\section{Variables and predicates}

- $v, u, u 1, u 2$ : variables denote a node belongs to the network.

- $\{U\}$ :set of nodes.

- good, bad, spe: states, a node can take one or two states at the same time, but not spe and bad or good and bad.

- $N_{x}(v)$ : the neighbor in the direction $x$ of the node $v: x \in\{(N),(E),(W),(N E),(S E) \operatorname{or}(N W)\}$.

- connected $_{v}$ : true if the node $v$ is connected to the network, false else (Boolean).

- State $_{v}(k)$ : the state of the node $v$, taking one or two of these values $k=$ good, bad or spe.

- $\operatorname{State}_{v}(s$, good $)$ : the node $v$ has $s$ ( $s$ an integer) neighbors that have the good state State (good).

- moveAroundgood $_{v}\left(u, P_{x}\right)$ : move around the neighbor $u$ in such a way that $u$ becomes a $v$ 's neighbor in the direction $x$ relative to $v$.

- Parent $(v, u)$ : the node $v$ is parent of node $u$.

- isLeaf $(v)$ : the node $v$ is a leaf in the tree.

Predicates checked only in the first round

1: Initiator $(v) \equiv\left(\neg N_{n w}(v)=\right) \wedge$ connected $_{v}$.

2: $\operatorname{State}_{v}($ bad $) \equiv$ connected $_{v} \wedge \neg$ Initiator $(v)$.

3: $\operatorname{State}_{v}($ good $) \equiv$ Initiator $(v)$.

4: $\operatorname{State}_{v}($ spe $) \equiv$ Initiator $(v)$.

\section{Predicates checked in each round}

5: $\operatorname{Parent}(v, v) \equiv$ Initiator $(v)$.

6: $\operatorname{Parent}(v, u) \equiv(\operatorname{Parent}(w, v), u \neq$

$w) \wedge$ neighbor $(v, u) \wedge$ State $_{u}($ bad $) \wedge(\nexists z \in$

$N(v)$, Parent $(v, z))$.

7: $\operatorname{isLeaf}(v) \equiv(\forall u \in$

$N(v), \neg$ Parent $(v, u) \wedge \neg$ Parent $(v, v))$.

8: (P1): $\operatorname{State}_{v}(\operatorname{good}) \equiv\left(N_{e}(v)=\right.$

$u \wedge$ State $_{u}($ good $\left.) \wedge \neg N_{n e}(u)\right) \vee$ State $_{v}(3$, good $) \vee$

$\left(\right.$ State $_{v}(2$, good $) \wedge\left(N_{n e}(v)=u \wedge\right.$ State $_{u}($ spe $\left.)\right) \vee$

$\left(N_{w}(v)=u \wedge\right.$ State $_{u}\left(\right.$ good $\left.\left._{)}\right)\right) \vee$ State $_{v}($ spe $)$.

9: $\operatorname{State}_{v}(s$, good $) \equiv\left(N_{x}(v)=\{U\},|U|=\right.$

$s \wedge$ State $_{\{u\}}($ good $\left.)\right)$.

10: (P1): $\operatorname{State}_{v}($ spe $) \equiv\left(N_{n w}(v)=\right.$

$u 1) \wedge\left(N_{n e}(v)=u 2\right.$, State $_{u 2}($ spe $\left.)\right)$.

11: (P2): $\operatorname{moveAround} \operatorname{bad}_{v}\left(u, P_{e}\right) \equiv i s L e a f(v) \wedge$

State $_{v}($ bad $) \wedge\left(N_{n w}(v)=u \wedge\right.$ State $_{u}($ bad $\left.)\right)$.

12: (P2): moveAround $b a d_{v}\left(u, P_{\text {se }}\right) \equiv$ isLeaf $(v) \wedge$

State $_{v}($ bad $) \wedge\left(N_{n e}(v)=u \wedge\right.$ State $_{u}($ bad $\left.)\right)$.

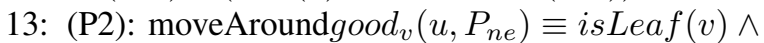

State $_{v}($ bad $) \wedge\left(N_{n w}(v)=u \wedge\right.$ State $_{u}($ good $\left.)\right)$.

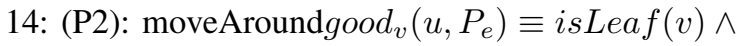

State $_{v}($ bad $) \wedge\left(N_{n e}(v)=u \wedge\right.$ State $_{u}($ good $\left.)\right)$.

the DASC Algorithm .

\section{B. Predicting the number of movements for each node}

To form the matrix of our square with $N \times N$ nodes, we begin with an incremental process with a single node that 
we assume in a correct square $1 \times 1$. After, we add each time a new layer contains the number of nodes of the last column plus the number of nodes of the last line of the current square plus one node. Considerer the figure 4 , the node $i$ will take a position $p+x$. Following the path from top to bottom the node $i$ will never move or move after all nodes after it, so if node $\mathrm{A}$ is before $\mathrm{B}, \mathrm{A}$ will take a position $p+c$, and node $\mathrm{B}$ will take a position $p+k$, with $c>k$. Adding layers, each time we add a new layer with number of nodes equal to the number of nodes of the previous layer plus two nodes, this can be expressed on the form of this numerical sequence:

$$
U_{j}=U_{j-1}+2
$$

Where: $U_{j}$ is the number of nodes in the layer $j$ and $U_{j-1}$ is the number of nodes in layer $j-1$.

In the chain we take a partitioning of the nodes to levels, a level can be associated to one or many nodes. The nodes take their levels with this following process: the first nodes that have $i \leq \sqrt{n}$ take the root level (level 0 ), for the other nodes, the first $x=(2 \sqrt{n}-2)$ nodes after the node $i=\sqrt{n}$ take the first level (level 1), and the second $x-2$ nodes take the second level and so on (figure 4 shows an example). So each node $i$ gets one level at the end.

The number of movements for each node $i$ of level $j$ can be given with the composition of two sequences $U_{i, j}$ and $R_{j}$.

$$
R_{j}=\left\{\begin{array}{r}
0, \text { if } j=0 \\
2 \sqrt{n}-5, \text { if } j=1 \\
R_{j-1}-2, \text { otherwise }
\end{array}\right.
$$

With $R_{j}$ is a number associated to nodes having the level $j$ and $n$ is network size.

$$
U_{i, j}=\left\{\begin{array}{r}
0, \text { if } i \leq \sqrt{n} \\
2, \text { if } i=\sqrt{n}+1, j=1 \\
U_{i-1}-R_{j}, \text { if } l(i+1) \neq j \\
U_{i-1}+2, \text { otherwise }
\end{array}\right.
$$

Where: $U_{i, j}$ and $U_{j}$ is the number of movements of node $i$ having level $j$ or the number of movements rounds of nodes having the level $j$ and $n$ is the network size.

Theorem 5.1: $n$ is highest number of movements in this algorithm.

Special case This case deals with the situation when the number of nodes is not a square root. We assume it $r$. To calculate the own movements we take a similar partitioning to the previous. In this special case also $r$ is the number of movements. The next lines are used to express the own movements for each node.

Let $n=\lfloor\sqrt{r}\rfloor$, and diff $=r-n^{2}$.

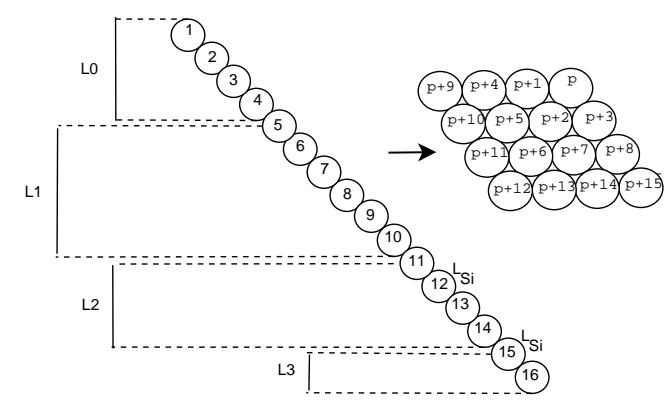

Figure 4. Example of nodes partitioning into levels and the final positions of nodes

$R_{j}$ has the same definition in (2).

$$
U_{i, j}=\left\{\begin{array}{r}
0, \text { if } i \leq n . \\
\operatorname{diff}+2 n-1, \text { if } i=n+1 . \\
U_{i-1}-2, \text { if } n+\operatorname{diff} \geq i>n . \\
\operatorname{diff}+2, \text { if } i=n+\operatorname{diff}+1 . \\
U_{i-1}-R_{j}, \text { if } i>n+\operatorname{diff}, l(i+1) \neq j . \\
U_{i-1}+2, \text { otherwise }
\end{array}\right.
$$

\section{Energy saving}

Sleeping state is used to save energy and awake state is used to do the task for each node. The node cannot enter to a sleep state by changing its state to good since the node after changing its state to good becomes a reference for neighbors or future neighbors and it should help its neighbors so they can take correct positions belong to the final shape. So, it should stay wake to send messages (consultation) to neighbor nodes that need to know its state to decide. The aim of the following functions is to find with an optimal and deterministic method the time slots when the node must wake up to help neighbors and where the node must sleep to save energy. The following functions have a form of mathematical sequences which are in fact messages. Thus, by receiving the information from its neighbor the node can know its value which refers to the time of entering wake or sleep state. We take a partitioning of nodes to levels, each node will have a level $l(i)$, and some nodes take special level say $l s_{i}$. Nodes with $i \leq \sqrt{n}$ take the level 0 . For the others nodes: the $x=2 \sqrt{n}-2$ nodes after $i=\sqrt{n}$ take level 1 . After, the following $x-2$ nodes take the following level (level 2) and so on. A special level $l s_{i}$ is associated to some nodes: node $4 \sqrt{n}-4$ takes the level $l s_{i}$, the following node that takes the level $l s_{i}$ is the one after $y=2 \sqrt{n}-5$, and the the following node that will take the level $l s_{i}$ is the one after $y-2$ nodes and so on. Figure 4 shows an example. Once the node has taken a child (in the tree) it may enter to the sleep state and it must wake up at time when it will have new neighbors, it is the time to reach it for nodes that are going up. The root node starts the building of the tree at the round $t 0$, it becomes a parent and enters into sleep state to save energy. Since the first leaf node will move $n$ 
rounds, (to become root's neighbor in the E direction) it will be neighbor of the root at the time $n-1$. So, the root adjusts the local clock to wake up at $n-1+O(n)$ (with $O(n))$ is the time the first tree) in order to collaborate with its new neighbors. Similarly, other nodes are waiting for the construction of the first tree and enter into sleep sate after having a child, each node located after $z$ nodes from the root enters into awake state at $n-z-1+O(n)$. The sequence $S_{i}$ expresses in term of $n$

the time when each node can enter into sleep state after helping its neighbors to take correct positions.

$$
T_{i}=\left\{\begin{array}{r}
7, \text { if } i=1 . \\
T_{i-1}+4, \text { otherwise }
\end{array}\right.
$$

Where : $T_{i}$ is a number associated to node $i, i \leq \sqrt{n}-2$.

$$
I_{j}=\left\{\begin{array}{r}
2 \sqrt{n}-1, \text { if } j=3 . \\
I_{j-1}-2, \text { otherwise }
\end{array}\right.
$$

Where : $I_{j}$ is a number associated to node $i$ has evel $j>1$.

$$
S_{i}=\left\{\begin{array}{r}
n+2, \text { if } i=1 . \\
S_{i-1}+T_{i-1}, \text { if } i \leq \sqrt{n}-1 . \\
S_{i-1}, \text { if } i=\sqrt{n} \vee i=\sqrt{n}+1 \\
S_{i-1}+2 \sqrt{n}-4, \text { if } l(i)=2 \wedge l(i-1)=1 . \\
S_{i-1}-2, \text { if } l s(i-1) .(E) \\
S_{i-1}-I_{i}, \text { if } l(i-1) \neq l(i) \\
S_{i-1}-1, \text { otherwise. }
\end{array}\right.
$$

Where : $S_{i}+O(n)$ refers to the sleeping time for node $i$.

\section{Generalization of the algorithm (DGASC)}

We have presented an algorithm that deals with one case of the chain, exactly with the case where nodes can have at the beginning neighbors in directions SE or NW or in both directions at the same time. To show how to generalize the algorithm in order to deal with any chain at the beginning it is important to show how to distinguishing the initiator (the root) whatever the case. For other nodes can know what form of chain is by looking at the direction of their two neighbors. The root can be distinguished with principle that it has only one neighbor in the direction SW or SE or E, obviously whatever the shape of the chain we cannot find one where another node that has only one neighbor in the direction $\mathrm{SW}$ or $\mathrm{SE}$ or $\mathrm{E}$, other nodes have two neighbors in the same time one in the direction $D$ and the other in the inverse direction say $-D$ for examples: one neighbor in $\mathrm{SE}$ direction and the other in the direction NW (NW-SE), SW and NE (NE-SW) or E and W(W-E). The last node in the chain has one neighbor in the direction NW, NE or W. After recognizing the form of chain, an algorithm similar to DASC presented is called, for example if the chain was with the form where the nodes can only have neighbors in the directions NE or SE or in both directions, we have to call $D A S C^{-d}$ if we define $D A S C^{-d}$ as the previous algorithm but the move is made from $\mathrm{NE}$ to $\mathrm{NW}$ or to $\mathrm{W}$ or from $\mathrm{NW}$ to $\mathrm{W}$.

\section{Simulation}

We have done the simulation with the declarative language Meld using DPRSim. In our simulations the radius of the node is $1 \mathrm{~mm}$. We simulated with a laptop with processor Intel(R) Core(Tm) i5, $2.53 \mathrm{Ghz}$. The results of these simulations come to agree the results obtained previously, in particular the number of movements for each node and the effectiveness of dynamicity. The nodes applied the procedure of partitioning to levels and predicted with the two functions $U_{i, j}$ and $R_{j}$ the number of movements for each node, at the end of the algorithm each node compares the results of prediction to the results calculated by it. The figure 6 represents the overall number of movements in the networks corresponds to

$$
O=\sum_{i=1, j=0}^{i=n, j=\sqrt{n}-1} U_{i, j}
$$

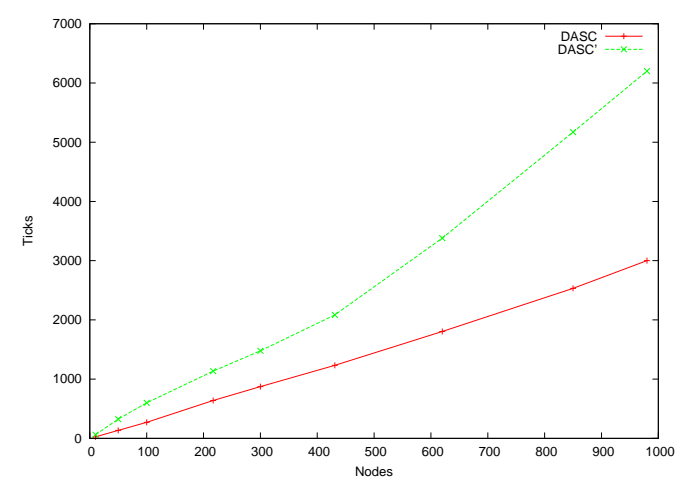

Figure 5. Execution time.

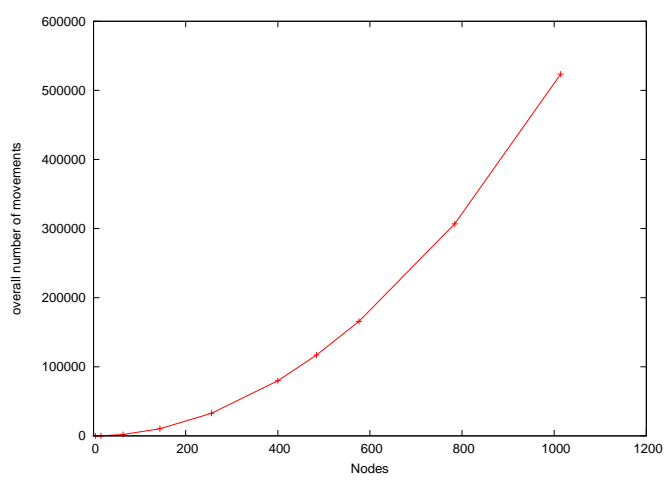

Figure 6. The overall number of movements in the network.

The nodes applied the procedure of nodes partitioning into levels and obtain with the function discussed previously the time slot when they enter into the sleep state and the wake 
up state. The figure 5 represents the execution time in ticks by the number of nodes, with counting the tree (DASC') and without counting the tree (DASC). In the curve representing the number of movements, we remark for some values of the network size $n$, the number is always $n$ as found in theory. For the curves that represents the execution time figure 5, without counting the time of construction of the tree of DASC we see that if the number of nodes increases the time increase. If we count the time of the tree $(O(n)$ time), the execution time of the algorithm increases dramatically. As conclusion, to ensure a Snap-connectivity through all time slots of the algorithm and to manage dynamically the nodes that can move, we have to use the tree, and by using the tree, we need more time to achieve the self-reconfiguration.

\section{CONCLUSION}

In this paper, we presented a new method to complete the self-reconfiguration where the nodes do not know the fixed positions of the target shape but only the aimed shape; nodes collaborate and help each other by analyzing the characteristics of the target shape. Compared to the literature works this algorithm is scalable because each node needs only three state to achieve the self-reconfiguration. Nodes in our paper can perform the algorithm regardless the place where they are because the algorithm is independent of the map, that what we call portability. We have presented a protocol that guarantees the connectivity throughout the execution time of the algorithm. The proposed algorithm is characterized by a constant memory needs and message exchange is limited to neighboring consultations. Consequently, system reconfiguration is fast. We presented how to manage the dynamicity of the network to save the energy and how to predict the movements of nodes in order to make the algorithm robust and energy-aware. However, some open problems remain; we will study the fault tolerance on self-reconfiguration in microrobots networks. The study of the effect of self-reconfiguration on the permutation routing where the objective will be to optimize the path of a node to go to the correct position where it finds its correct data. Also, the use of tabu algorithms to achieve the self-reconfiguration.

\section{ACKNOWLEDGMENTS}

This work is funded by the Labex ACTION program (contract ANR-11-LABX-01-01), ANR/RGC (contracts ANR12-IS02-0004-01 and 3-ZG1F) and ANR (contract ANR2011-BS03-005). The authors wish to express their appreciation to the anonymous reviewers for their constructive comments.

\section{REFERENCES}

[1] M. P. Ashley-Rollman, S. C. Goldstein, P. Lee, T. C. Mowry, and P. Pillai, Meld: A Declarative Approach to Programming Ensembles, In Proceedings of the IEEE International Conference on Intelligent Robots and Systems (IROS '07), October, 2007.
[2] M. P. Ashley-Rollman, P. Lee, S. C. Goldstein, Padmanabhan Pillai, and Jason D. Campbell, A Language for Large Ensembles of Independently Executing Nodes, In Proceedings of the International Conference on Logic Programming, July, 2009.

[3] J. Bourgeois and S.C. Goldstein. Distributed Intelligent MEMS: Progresses and perspectives, 3-rd Int. Conf. ICT Innovations, volume of Advances in Intelligent and Soft Computing, pages 15-25, Ohrid, Macedonia, September 2012.

[4] D. Dewey, S. S. Srinivasa, M. P. Ashley-Rollman, M. D. Rosa, P. Pillai, T. C. Mowry, J. D. Campbell, and S. C. Goldstein, Generalizing Metamodules to Simplify Planning in Modular Robotic Systems, In Proc. of IEEE/RSJ International Conference on Intelligent Robots and Systems, September, 2008.

[5] J. Eckert, and H. Lichte, and F. Dressler and H. Frey, On the Feasibility of Mass-Spring-Relaxation for Simple SelfDeployment, 8th IEEE/ACM International Conference on Distributed Computing in Sensor Systems Hangzhou, China, 2012.

[6] C. Jones, M. J. Mataric, From local to global behavior in intelligent self-assembly.In: Proc. 2003 IEEE International Conference on Robotics and Automation,, vol. 1, pp. 721-726. IEEE Computer Society Press, Los Alamitos, 2003.

[7] S. Jeon, C. Ji, Randomized Distributed Configuration Management of Wireless Networks: Multi-layer Markov Random Fields and Near-Optimality CoRR abs/0809.1916, 2008.

[8] F. Kribi, P. Minet, A. Laouiti, Redeploying mobile wireless sensor networks with virtual forces, IFIP Wireless Days, Paris, France, December 2009.

[9] H. Lakhlef, H. Mabed, J. Bourgeois, Distributed and Efficient Algorithm for Self-reconfiguration of MEMS Microrobots, in the 28th ACM Symposium On Applied Computing, pages 560-566, Coimbra, Portugal, March 2013.

[10] H. Lakhlef, H. Mabed, J. Bourgeois, Parallel Selfreconfiguration for MEMS Microrobot, in the 7-th IEEE Region 8 International conference on Computer as a Tool, Zagreb, Croatia, July 2013.

[11] H. Mabed, H. Lakhlef, J. Bourgeois Fully Distributed Redeployement Algorithm for Multi-Robot System.In: 6th Int. Conf. on NETwork Games, COntrol and OPtimization, NetGCooP'12. IEEE Computer Society, Avignon, France, 2012.

[12] M. Mamei, A. Roli, F. Zambonelli, Emergence and Control of Macro Spatial Structures in Perturbed Cellular Automata, and Implications for Pervasive Computing Systems, IEEE Transactions on Systems, Man, and Cybernetics, 36(5), May 2005.

[13] M. Mamei, M. Vasirani, F. Zambonelli, Experiments of Morphogenesis in Swarms of Simple Mobile Robots, Journal of Applied Artificial Intelligence, 8(9-10):903-919, Oct. 2004.

[14] D. Rus, M. Vona, Crystalline robots: Self-reconfiguration with compressible unit modules, Autonomous Robots 10(1), 107-124, 2001.

[15] K. Stoy, R. Nagpal, Self-Repair Through Scale Independent Self-Reconfiguration, Proc. IEEE/RSJ International Conference on Intelligent Robotsn and systems, Sendai, japan, 2004.

[16] B. Warneke, M. Last, B. Leibowitz, and K.S.J Pister,K.S.J., 2001, Smart Dust: Communicating with a Cubic-Millimeter Computer, Computer Magazine, pp. 44-51, 2001.

[17] P. White, V. Zykov, J. C. Bongard, H. Lipson, Three dimensional stochastic reconfiguration of modular robots In: Proceedings of Robotics Science and Systems, pp. 161-168. MIT Press, Cambridge , 2005.

[18] Physical rendering simulator (dprsim): http://www.pittsburghintel-research.net/dprweb. 